\title{
Wurst und Schinken sollen Asthma verstärken
}

\author{
Gepökelte Fleischerzeugnisse sollten nicht allzu oft auf dem Speiseplan von Asthmapatienten \\ stehen: Einer französischen Studie zufolge besteht, unabhängig vom Gewicht, ein Zusammen- \\ hang mit der Zunahme von Asthmabeschwerden.
}

\begin{abstract}
Regelmäßig gepökelte Fleischerzeugnisse zu essen, gilt in vielerlei Hinsicht als gesundheitsschädlich: Die WHO hat die Produkte letztes Jahr als karzinogen eingestuft. Das Risiko für chronische Erkrankungen wie KHK und Diabetes ist erhöht.

Auch zu Lungenschäden, COPD(-Exazerbationen) und Lungenkrebs werden Verbindungen gesehen. Laut einer prospektiven Studie aus Frankreich sind Asthmapatienten, die reichlich Wurst und Schinken essen, häufiger von einer Verschlechterung betroffen (Thorax 2016, online 20. Dezember). Da Übergewicht das Risiko von Asthma(exazerbationen) erhöht, besteht die besondere Herausforderung einer Studie zu Ernährung und Asthma darin, den Einfluss des BMI korrekt zu berücksichtigen. Wird der BMI als Störfaktor betrachtet und entsprechend adjustiert, besteht die Gefahr einer „Überadjustierung“, falls der BMI ein vermittelnder Faktor ist. Die Autoren dieser Studie haben daher Mediationsanalysen vorgenommen, mit denen direkte und indirekte Effekte voneinander getrennt werden können.

\section{Kohortenstudie als Grundlage}

Die zugrunde liegenden Daten stammen aus einer Kohorten-
\end{abstract} studie (EGEA), an der erwachsene Asthmapatienten, erstgradige Verwandte und Kontrollpersonen beteiligt waren; $42 \%$ von ihnen litten an Asthma. In den Jahren 2003 bis 2007 beantworteten 971 Teilnehmer ausgedehnte Ernährungsfragebogen. Der Verzehr von Wurst und Schinken wurde im Median mit 2,5 Portionen pro Woche beziffert (eine Portion entsprach etwa zwei Scheiben). Während der Nachbeobachtungszeit von im Mittel sieben Jahren kam es bei $20 \%$ der Teilnehmer zu einer Zunahme von Asthmabeschwerden wie Engegefühl in der Brust oder Atemnot in verschiedenen Situationen.

Der Anteil der Personen mit einer Symptomverschlechterung lag je nach Konsum von Wurst und Schinken - also $<1,1-3,9$ oder $\geq 4$ Portionen pro Woche - bei $14 \%$, $20 \%$ oder $22 \%$. Jene, die die meisten gepökelten Fleischerzeugnisse aßen, waren allerdings auch häufiger Raucher und ernährten sich insgesamt kalorienreicher und mit mehr Natrium und mehr gesättigten Fettsäuren. Aber auch nach Adjustierung für diese Risikofaktoren sowie für Alter, Geschlecht und körperliche Aktivität ergab sich in der Mediationsanalyse ein direkter Effekt von gepökelten Fleischprodukten auf die Asthmasymptomatik: Das Risiko für eine Verschlechterung lag in der Gruppe mit dem höchsten Verzehr um $76 \%$ höher als in der Gruppe mit dem geringsten.

Zusätzlich ließ sich ein indirekter Effekt, vermittelt über den Anstieg des BMI, feststellen. Hier betrug der Risikozuwachs aber nur $7 \%$. Damit waren vom Gesamteffekt des Fleischprodukteverzehrs nur $14 \%$ auf den BMI zurückzuführen.

\section{Wintersport ist für Asthmatiker besonders günstig}

Trockene und kalte Luft belastet die Atemwege. Trotzdem brauchen Asthmatiker nicht auf Wintersport zu verzichten. „Asthmatiker, die richtig mit ihrer chronischen Atemwegskrankheit umgehen, können auch Skifahren und anderen Wintersport betreiben", so Dr. Gerd Schauerte, Pneumologe und Allergologe im CJD Berchtesgaden - Asthmazentrum.

Studiendaten deuten darauf hin, dass Asthmatiker, die im Winter Sport treiben, davon sogar besonders profitieren. Günstig für Asthmatiker ist beim Sport eine intervallartige Belastung mit Erholungspausen dazwischen. Das schützt vor einem Anfall. Bei Skiabfahrten legen die meisten immer wieder kleine Pausen ein, und dadurch ergibt sich automatisch der gewünschte Wechsel. „Deshalb ist Skifahren ganz günstig“, so Schauerte. Nur wenn die Temperaturen zu tief in den Keller gehen, sei

Vorsicht geboten. Bei unter $-10^{\circ} \mathrm{C}$ sollte man sich nicht unbedingt auf Ski oder Snowboard stellen. Das gilt besonders für Skilanglauf oder Schneeschuhwanderungen. Für diese Ausdauerbelastungen ist eine Prämedikation sinnvoll, bei der der Asthmatiker vor dem Sport sein Asthmaspray inhaliert. Für Schauerte ist klar: „Asthmatiker sollten möglichst kontinuierlich Sport machen - gerade auch im Winter."

Quelle: Pressemitteilung der CJD Berchtesgaden vom 31.01.2017 